\title{
The quark flavor problem and spontaneous breaking of flavour $S U(3)^{3}$
}

\author{
E. Nardi* \\ INFN-Laboratori Nazionali di Frascati \\ C.P. 13, I-00044 Frascati, Italy \\ E-mail: enrico.nardi@lnf.infn.it
}

A $S U(3)_{Q} \times S U(3)_{u} \times S U(3)_{d}$ invariant scalar potential breaking spontaneously the quark flavour symmetry can explain the Standard Model flavour puzzle. The approximate alignment in flavour space of the vacuum expectation values of the up and down 'Yukawa fields' results as a dynamical effect. The observed quark mixing angles, the weak $\mathrm{CP}$ violating phase, and hierarchical quark masses can be all reproduced at the cost of introducing additional (auxiliary) scalar multiplets, but without the need of introducing hierarchical parameters.

18th International Conference From the Planck Scale to the Electroweak Scale 25-29 May 2015

Ioannina, Greece

*Speaker. 


\section{Introduction}

Fermion family replication represents probably the oldest unexplained puzzle in elementary particle physics, dating back to the discovery of the muon by Anderson and Neddermeyer at Caltech in 1936. With the discovery second and third generation particles, the puzzle became even more intriguing: fermions with the same $S U(3)_{C} \times S U(2)_{L} \times U(1)_{Y}$ quantum numbers have been found with mass values that span up to five orders of magnitude. Explaining such strongly hierarchical mass patterns requires a more fundamental theory than the Standard Model (SM), and a plethora of attempts in this direction have been tried. In the large majority they follow two types of approaches:

(i) The first is to postulate new symmetries under which fermions with the same SM quantum numbers transform differently. The fact that fermion families appear to replicate is then just an illusory feature of the low energy theory, ascribable to our incomplete knowledge of the full set of fundamental quantum numbers. This is, for example, the basic ingredient of the popular Froggatt-Nielsen mechanism [1], in which the hierarchy of the Yukawa couplings follows from a dimensional hierarchy in the corresponding effective Yukawa operators, obtained by assigning to the lighter generations larger values of new Abelian charges.

(ii) A different approach is to assume that the three generations contain exact replica of the same states. For each fermion type (same charge and chirality) the gauge invariant kinetic term is characterized by a $U(3)$ (flavour) symmetry [2]. When this symmetry is broken explicitly by the Yukawa terms we have the SM. A more interesting idea is that the flavour symmetry is broken spontaneously (SFSB) by vacuum expectation values (vevs) of 'Yukawa fields' with transformations under the flavour group such that at the Lagrangian level, the flavour symmetry is exact.

The first approach relies on ad hoc assignments of new quantum numbers in order to reproduce qualitatively the observed mass patterns. The second approach can be considered theoretically more ambitious (as it relies on less ad hoc assumptions) although it is by far more challenging than the first one regarding successful model implementations. In order to offer a natural solution to the Yukawa hierarchy, such models should not rely on a hierarchical arrangement of parameters or on some tuning between them. Loop-induced hierarchies for example would be plausible [3], but we have found that this possibility is vetoed [4]. Dynamical mechanisms inducing strong suppression of some parameters are another possibility which we have instead proved being viable [5].

The idea that quark masses could arise from the minimum of a scalar potential invariant under a suitable symmetry is rather old, and group theoretical methods to identify the natural extrema of a 'Yukawa potential' were established already in the early seventies [6, 7]. Nowadays the literature on attempts to explain dynamically the Yukawa couplings (employing different flavour groups and different flavour-breaking fields) is extensive $[8,9,10,11,12,13,14,15,16,17,18,19,20]$.

This contribution is based on three papers $[3,4,5]$. It resumes the main steps (difficulties, vetoes, mandatory requirements) that lead us to understand the features needed to implement SFSB in what we think is the best motivated scenarios, which is based on the quark flavour symmetry: $\mathscr{G}_{\mathscr{F}}=S U(3)_{Q} \times S U(3)_{u} \times S U(3)_{d}$, where $Q,(u$ and $d)$ denote the $S U(2)_{L}$ quark doublets (singlets). Quarks couple to the Higgs field via the effective operator

$$
-\mathscr{L}_{Y}=\sum_{q=u, d}\left[\frac{1}{\Lambda} \bar{Q} Y_{q} q H_{q}+\text { h.c. }\right],
$$


where $H_{d}=H$ is the Higgs field $\left(H_{u}=i \sigma_{2} H^{*}\right), Y_{u, d}$ are the up- and down-type Yukawa fields, and $\Lambda$ is the scale where the effective operators arise. The theoretical challenge is now finding a

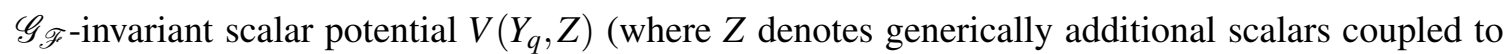
$Y_{q}$ in a symmetry invariant way) which can break $\mathscr{G}_{\mathscr{F}}$ spontaneously and yield a set of vevs $\left\langle Y_{q}\right\rangle$ reproducing the observed structure of the SM Yukawa couplings.

\section{A single Yukawa field: generating a hierarchy}

Let us start by considering a single Yukawa multiplet, e.g. $Y=Y_{u}$, that under the relevant flavour symmetry $\mathscr{G}_{L R}=S U(3)_{L} \times S U(3)_{R}$ transforms as $(3, \overline{3})$. As a first step, we want to explore the possibility of generating a Yukawa hierarchy via SFSB of $\mathscr{G}_{L R}$.

\subsection{Minimization of the tree level potential}

It is convenient to parameterize $Y$ by means of its singular value decomposition:

$$
Y=\mathscr{V}^{\dagger} \chi \mathscr{U}, \quad \chi=\operatorname{diag}\left(u_{1}, u_{2}, u_{3}\right),
$$

where the matrices $\mathscr{V}$ and $\mathscr{U}$ are unitary and the entries in $\chi$ are real nonnegative. We can write down three renormalizable invariants with respect to the $\mathscr{G}_{L R}$ transformations $Y \rightarrow V_{L} Y V_{R}^{\dagger}$ :

$$
\begin{aligned}
& T=\operatorname{Tr}\left(Y Y^{\dagger}\right)=\sum_{i} u_{i}^{2}, \\
& A=\operatorname{Tr}\left[\operatorname{Adj}\left(Y Y^{\dagger}\right)\right]=\frac{1}{2} \sum_{i \neq j} u_{i}^{2} u_{j}^{2}, \\
& \mathscr{D}=\operatorname{Det}(Y)=e^{i \delta} \prod_{i} u_{i} \equiv e^{i \delta} D,
\end{aligned}
$$

where $\delta=\operatorname{Arg}\left[\operatorname{Det}\left(\mathscr{V}^{\dagger} \mathscr{U}\right)\right]$ and $D=|\mathscr{D}|$. The most general potential reads:

$$
V=\lambda\left[T-\frac{m^{2}}{2 \lambda}\right]^{2}+\tilde{\lambda}_{A} A+\tilde{\mu} \mathscr{D}+\tilde{\mu}^{*} \mathscr{D}^{*},
$$

and by taking $\tilde{\mu}=\mu e^{i \phi}$ the last two terms can be also rewritten as $2 \mu \cos (\phi+\delta) D$. For the rest of this section we will neglect the phases of $\mu$ and $\mathscr{D}$ and assume that both quantities are real. Formally, one can implement this condition in the scalar potential via a chiral rotation of the quark fields in eq. (1.1). This is described in detail in [21] (although it was later found that the solution to the strong CP problem put forth in [21] does not hold [5], the analysis of the relation between the phase $\phi+\delta$ chiral rotations and the chiral anomaly remains valid). In eq. (2.5) we require $\lambda>0$ in order to have a potential bounded from below and $m^{2}>0$ to trigger SSB via $\langle T\rangle \neq 0$. Eqs. (2.3) and (2.4) show that $A$ and $D$ are maximized for the symmetric configuration $\langle\chi\rangle_{s}=\left(u_{s}, u_{s}, u_{s}\right)$. This would break $\mathscr{G}_{L R}$ to the maximal subgroup $H_{S}=S U(3)_{L+R}$. The minimum value $\langle D\rangle=0$ is obtained when one entry in $\langle\chi\rangle$ vanishes, while $\langle A\rangle=0$ is obtained when two entries vanish: $\langle\chi\rangle_{h}=\left(0,0, u_{h}\right)$ (with $u_{h}=m / \sqrt{2 \lambda}$ ). Therefore, if $\lambda_{A}>0$ and a specific condition $\mu^{2} / m^{2}<F\left(\lambda, \lambda_{A}\right)$ is satisfied (where $F\left(\lambda, \lambda_{A}\right)$ is a simple algebraic expression of its arguments, see [3]) the global minimum is obtained for $\langle\chi\rangle_{h}$. This gives as little group the maximal $\mathscr{G}_{\mathscr{F}}$ subgroup $H_{h}=S U(2)_{L} \times S U(2)_{R} \times U(1)$ [7]. 


\subsection{The one loop effective potential}

The fact that the tree level potential admits the vacuum configuration $\langle\chi\rangle_{h}=\left(0,0, u_{h}\right)$ which is a good approximation to the quarks Yukawa pattern, is encouraging, and one could hope that some type of corrections could lift the zeroes to hierarchically suppressed entries. Clearly this would correspond to breaking further the little groups of the tree level vacua. That is, we need to understand if a stepwise breaking $\mathscr{G}_{L R} \rightarrow H_{h} \rightarrow$ nothing, of the initial symmetry group, is possible. In [3] it was shown that by adding to the tree level potential new terms of the form $c_{D} \mu D \log D$ and $c_{A} A \log A$, with coefficients $c_{D, A}<1$, would do exactly this, lifting the two zeroes to nonvanishing but exponentially suppressed entries. In the same paper it was conjectured that the one loop corrected effective potential might contain precisely terms of this type. The non trivial task of computing the effective potential for the scalar multiplet $Y$ was undertaken in [4]. We first worked out the analytical expression of $V_{\text {eff }}$, and next we carried out a numerical study of its minima. We have found that the vacuum structure of the tree level minimum remains stable against loop corrections. The reason for this stability can in fact be understood on the basis of a theorem established by Georgi and Pais (GP) in the late seventies [22], stating that: stepwise SSB can only occur via perturbative quantum corrections if there are non-Goldstone massless bosons in the tree approximation. Since in our case all massless modes correspond to Goldstone bosons, the little groups $H_{s, h}$ cannot undergo further breaking because of loop effects. (The Coleman-Weinberg model [23] is a well known example of a tree level symmetry broken by loop effects. This can occur because classical scale invariance of the scalar potential is assumed, and thus at the tree level all the scalars are massless non-Goldstone modes. Therefore the occurrence of loop induced breaking is in agreement with the GP theorem.) In ref. [4] the GP theorem was extended to include also the case of perturbations due to effective operators involving higher order invariants of $Y$, and this lead to the conclusion that, in the absence of tree level non-Goldstone massless modes: no perturbative effect of any kind can further break the tree level little groups $H_{s, h}$ and lift the vanishing entries in $\langle\chi\rangle$. As a side remark, it should be noticed that the previous result does not mean that one cannot write a polynomial expression in the invariants which, upon minimization, yields a hierarchical pattern $\langle\chi\rangle_{\exp }=\left(\varepsilon^{\prime}, \varepsilon, u\right)$ $\left(\varepsilon^{\prime} \ll \varepsilon \ll u\right)$ and breaks $\mathscr{G}_{\mathscr{F}}$ completely. This in fact can be done straightforwardly. For a given type of quarks, let us denote the experimental value of the invariants as $T_{\exp }, A_{\exp }, D_{\exp }$. Then the nonnegative polynomial $P(Y)=\left(T-T_{\exp }\right)^{2}+\frac{1}{\Lambda^{4}}\left(A-A_{\exp }\right)^{2}+\frac{1}{\Lambda^{2}}\left(D-D_{\exp }\right)^{2}$ is guaranteed to have its minimum in $\langle\chi\rangle_{\exp }$ [16]. (Of course, with a suitable set of independent invariants, this can be extended to the full quark sector, so that all quark masses and mixing angles can be trivially obtained via minimization of a suitable polynomial.) However, expanding $P(Y)$ and truncating retaining only the renormalizable operators, one obtains that the $A$ invariant appears with an overall negative sign $-2\left(A_{\exp } / \Lambda^{4}\right) A$ and this implies that the minimum occurs for the symmetric solution $\langle\chi\rangle_{s}=\left(u_{s}, u_{s}, u_{s}\right)$. Thus the configuration $\langle\chi\rangle_{\exp } \sim\left(\varepsilon^{\prime}, \varepsilon, u\right)$ is not obtained from a perturbation of the tree level vacuum $\langle\chi\rangle_{h} \sim\left(0,0, u_{t}\right)$, implying that higher order terms dominate over the renormalizable ones. Thus, such an approach would result in an incurable loss of predictivity.

\subsection{Symmetry breaking via reducible representations}

The results of the previous section make clear which way is left open to get a phenomenologically viable pattern of vevs for the components of the Yukawa field $Y$. Namely, the flavour sym- 
metry $\mathscr{G}_{L R}$ must be completely broken already at the tree level. For this, we need a non-minimal set of scalar fields in reducible representations of the flavour group. In fact, breaking a symmetry by means of reducible representations avoids at once the issue of stability of the little groups that are maximal subgroup of the original symmetry. A minimal enlargement of the scalar sector involves adding two multiplets, $Z_{L, R}$ transforming respectively in the fundamental of one of the two group factors $S U(3)_{L} \times S U(3)_{R}$, while being singlets under the other one: $Z_{L}=(\mathbf{3}, \mathbf{1})$ and $Z_{R}=(\mathbf{1}, \mathbf{3})$. Let us write the most general $S U(3)_{L} \times S U(3)_{R}$ invariant potential for $Z_{L}, Z_{R}$ and $Y=(\mathbf{3}, \overline{\mathbf{3}})$ as:

$$
V\left(Y, Z_{L}, Z_{R}\right)=V_{\mathscr{I}}+V_{\mathscr{A} \mathscr{R}}+V_{\mathscr{A}} .
$$

$V_{\mathscr{I}}$ collects the so called flavour irrelevant terms [5]. These are terms that are invariant under accidental symmetries that are much larger than the flavour symmetry, and thus the values of their vevs do not depend on any particular flavour configuration. For example $T$ eq. (2.2) is invariant under $S O(18)$ which is broken down to $S O(17)$ after SFSB. A transformation of this large subgroup can rotate $\langle\chi\rangle_{s}$ into $\langle\chi\rangle_{h}$ leaving unchanged the "length" of the vev of $Y$ (defined as $\sqrt{\langle T\rangle}$ ). Another example of flavour irrelevant operators is $\left|Z_{L, R}\right|^{2}$ which carries a $S O(6)$ symmetry. All in all, $V_{\mathscr{I}}$ just determines the "length" of the vevs for $Y, Z_{L}, Z_{R}$, without contributing to determine any specific flavour direction. Therefore we will omit writing here its explicit form, that can be found in [4].

Those terms in the potential that tend to break the symmetry to the largest maximal little group (in this case $S U(3)_{L+R}$ with eight generators) are defined as attractive $(\mathscr{A})$, while terms that tend to break the symmetry to the smallest maximal little group (in this case $S U(2)_{L} \times S U(2)_{R} \times U(1)$ with seven generators) are called repulsive $(\mathscr{R})$. Hermitian monomials can be attractive or repulsive depending if their (real) couplings are negative or positive, and are included in $V_{\mathscr{A} \mathscr{R}}$ :

$$
V_{\mathscr{A} \mathscr{R}}=\lambda_{A} A+g_{R}\left|Y Z_{R}\right|^{2}+g_{L}\left|Y^{\dagger} Z_{L}\right|^{2}
$$

where we have adopted for the modulus square notation the convention $|X|^{2}=X^{\dagger} X$. For example (see section 2.1) the sign of $\lambda_{A}$ determines if the $A$ invariant is attractive or repulsive. Operators which correspond to non-Hermitian monomials are included in $V_{\mathscr{A}}$ :

$$
V_{\mathscr{A}}=\tilde{\mu} \mathscr{D}+\tilde{v} Z_{L}^{\dagger} Y Z_{R}+\text { H.c. }=2 \mu D \cos \delta+2 v\left|Z_{L}^{\dagger} Y Z_{R}\right| \cos \phi_{L R},
$$

where in the second equality $\mu=|\tilde{\mu}|$ and $v=|\tilde{v}|$. Non-Hermitian monomials are always attractive, as is the case for $\mathscr{D}$ : when the vev $\langle\mathscr{D}\rangle$ is nonvanishing, minimization drives its phase $\delta \rightarrow \pi$ $(\cos \delta=-1)$. Then the minimum gets lowered for the largest possible value of $D$, that is obtained for $\langle\chi\rangle_{s}$, corresponding to the largest little group $H_{s}=S U(3)_{L+R}$. Let us now define

$$
\langle Y\rangle=v_{Y} \operatorname{diag}\left(\varepsilon^{\prime}, \varepsilon, y\right), \quad\left\langle Z_{L}\right\rangle=v_{L}\left(z_{L}, \varepsilon_{L}^{\prime}, \varepsilon_{L}\right), \quad\left\langle Z_{R}\right\rangle=v_{R}\left(z_{R}, \varepsilon_{R}^{\prime}, \varepsilon_{R}\right)
$$

with $\varepsilon^{2}+\varepsilon^{\prime 2}+y^{2}=1$ and analogously for the entries in $\left\langle Z_{L, R}\right\rangle$. Let us see if a hierarchical solution $\varepsilon, \varepsilon^{\prime} \ll y, \varepsilon_{L, R}, \varepsilon_{L, R}^{\prime} \ll z_{L, R}$, can be obtained. $V_{\mathscr{I}}$ in eq. (2.6) fixes the 'lengths' $v_{Y}$ and $v_{L, R}$, and vanishes, so we need to consider only the effect of $V_{\varepsilon} \equiv V_{\mathscr{A} \mathscr{R}}+V_{\mathscr{A}}$. For $\lambda_{A}, g_{L, R}>0 V_{\mathscr{A} \mathscr{R}}$ is always positive, and thus it is minimized when it vanishes, which occurs when the vevs of $Z_{L, R}$ are misaligned with respect to the vev of $Y$, as for example $\langle Y\rangle=v_{Y} \operatorname{diag}(0,0,1),\left\langle Z_{L}\right\rangle=v_{L}\left(c_{L}, s_{L}, 0\right)$ and $\left\langle Z_{R}\right\rangle=v_{R}\left(c_{R}, s_{R}, 0\right)\left(c_{L, R}^{2}+s_{L, R}^{2}=1\right)$. However, such a configuration would also imply $V_{\mathscr{A}}=0$, 
while configurations yielding $V_{\mathscr{A}}<0$ would result in a lower minimum. We then learn that the parameters $\mu$ and $v$ appearing in $V_{\mathscr{A}}$ can play a crucial role in lifting the vanishing entries. A simple illustrative example of their action can be given by setting for simplicity $v_{Y}=v_{L}=v_{R}$ and $\lambda_{A}=g_{L}=g_{R}$. Solving for the extremal conditions $\partial V_{\varepsilon} / \partial \varepsilon=\partial V_{\varepsilon} / \partial \varepsilon^{\prime}=\partial V_{\varepsilon} / \partial \varepsilon_{L, R}=\partial V_{\varepsilon} / \partial \varepsilon_{L, R}^{\prime}=$ 0 , and truncating to terms $\mathscr{O}\left(\varepsilon^{2}\right)$ we obtain a unique solution for the global minimum:

$$
\varepsilon=\frac{\lambda_{A} v v_{Y}}{3 \lambda_{A}^{2} v_{Y}^{2}-\mu^{2}}, \quad \varepsilon^{\prime}=\frac{\mu}{\lambda_{A} v_{Y}} \varepsilon
$$

At this order, the other parameters vanish $\left(\varepsilon_{L, R}=\varepsilon_{L, R}^{\prime}=0\right)$, and the potential minimum is $V_{\varepsilon}^{\min }=$ $-v v_{Y}^{3} \varepsilon$. Eq. (2.10) shows that a hierarchy $\varepsilon^{\prime} \sim 10^{-2} \varepsilon \sim 10^{-4}$, which would fit well the observed values of $\left\langle\chi_{u}\right\rangle$, can be obtained by taking $v \sim \mu \sim 10^{-2} \lambda_{A} v_{Y}$.

\section{Two Yukawa fields: generating quark mixing}

In this section we extend the study of SFSB by including both the $u$ and $d$ sectors. The flavour symmetry is then the full flavour group $\mathscr{G}_{\mathscr{F}}$ under which the $u$ - and $d$-type Yukawa multiplets transform as: $Y_{u} \sim(\mathbf{3}, \overline{\mathbf{3}}, \mathbf{1})$ and $Y_{d} \sim(\mathbf{3}, \mathbf{1}, \overline{\mathbf{3}})$.

\subsection{Minimization of the potential for $Y_{u}$ and $Y_{d}$}

With just the two multiplets $Y_{u}$ and $Y_{d}$ we can write only one invariant that is not flavour irrelevant and that couples the two Yukawa fields (for the complete expression for $V\left(Y_{u}, Y_{d}\right)$ see [3]):

$$
T_{u d}=\operatorname{Tr}\left(Y_{u} Y_{u}^{\dagger} Y_{d} Y_{d}^{\dagger}\right)=\operatorname{Tr}\left(K^{\dagger} \chi_{u}^{2} K \chi_{d}^{2}\right)
$$

where $K$ is a unitary matrix of fields that in terms of the singular value decomposition parameterization eq. (2.1) is given by $K=\mathscr{V}_{u} \mathscr{V}_{d}^{\dagger}$. The vev of $K$ describes the mismatch between the two basis in which $\left\langle Y_{u}\right\rangle$ and $\left\langle Y_{d}\right\rangle$ are diagonal, and after ordering $\left\langle\chi_{u}\right\rangle$ and $\left\langle\chi_{d}\right\rangle$ in the same way (e.g. with increasing size of their entries) we can make the identification $\langle K\rangle=V_{C K M}$. Being a Hermitian monomial, $T_{u d}$ can be attractive or repulsive depending on the sign of its coupling $\lambda_{u d}$ (respectively negative or positive). At fixed lengths $\left(\sqrt{\left\langle T_{u}\right\rangle}, \sqrt{\left\langle T_{d}\right\rangle}=\right.$ const.) when $\left\langle Y_{u}\right\rangle$ and $\left\langle Y_{d}\right\rangle$ are "aligned" (i.e. they are diagonal in the same basis and with the same ordering) the value of $\left\langle T_{u d}\right\rangle$ is maximum, while it is minimum when in a given basis they are "anti-aligned" (diagonal but with opposite ordering). It is then clear that there are only two options to extremize $T_{u d}$ : if its coupling $\lambda_{u d}$ is negative, alignment is selected and we obtain $\langle K\rangle=I . \lambda_{u d}>0$ selects instead the anti-aligned configuration, which means that $\langle K\rangle$ is anti-diagonal. The first possibility can be considered to give a reasonable first approximation to $V_{C K M}$. However, also in this case there is no type of perturbative correction that can produce departures from an exact $\langle K\rangle=I$ and generate small mixing angles. A simple intuitive way to understand this is to notice that with only two "directions" in flavour space $\left(Y_{u}\right.$ and $\left.Y_{d}\right)$ there is just a single relative "angle", which gets fixed by minimization of the potential. This implies that alignment or anti-alignment are the only possibilities for extremization.

One additional remark is in order. With a negative coupling $\left(\lambda_{u d}<0\right)$ the term in eq. (3.1) besides aligning $\left\langle Y_{u}\right\rangle$ and $\left\langle Y_{d}\right\rangle$ has also another important effect: the naive hierarchical pattern $y \sim 1$, $\varepsilon \sim \frac{v}{v_{Y}}, \varepsilon^{\prime} \sim \frac{\mu}{v_{Y}} \varepsilon$ that we have derived at the end of section 2.3, see eq. (2.10), gets amplified by the effect of $T_{u d}$. Numerically, we find that in the presence of $T_{u d}$ and with $\lambda_{u d}<0, \frac{\mu}{v} \sim 10^{-1}\left|\lambda_{u d}\right|$ is 
enough to generate sufficiently strong Yukawa hierarchies as the ones observed. For an explanation of how this dynamical enhancement comes about we refer to [5].

\subsection{Quark mixing via reducible representations}

As we have seen in section 2.3, adding new scalar representations is in any case necessary to generate hierarchical Yukawa matrices with nonvanishing entries. We will now argue that this can also cure the troublesome result $V_{C K M}=I$. The pair of scalar multiplets $Z_{L, R}$ transforming respectively in the fundamental of the $S U(3)_{L, R}$ factors of $\mathscr{G}_{L R}$ suffices to generate hierarchical entries for one Yukawa multiplet. Then, as a first attempt to generate a vev $\langle K\rangle$ with a non trivial structure, we introduce as a minimal number of additional fields, the following three multiplets: $Z_{Q_{1}} \sim(\mathbf{3}, \mathbf{1}, \mathbf{1})$, $Z_{u} \sim(\mathbf{1}, \mathbf{3}, \mathbf{1})$ and $Z_{d} \sim(\mathbf{1}, \mathbf{1}, \mathbf{3})$, where in parenthesis we have given the transformation properties under $\mathscr{G}_{\mathscr{F}}$. Therefore we have in total three 'vectors' $Z_{Q_{1}}, Y_{u}$ and $Y_{d}$ transforming under the L-handed factor $S U(3)_{Q}$ of $\mathscr{G}_{\mathscr{F}}$, while $Z_{u}$ and $Z_{d}$ transform respectively in the fundamental of the two R-handed factors. The study of the most general tree level potential for these five multiplets in terms of attractive/repulsive operators is a bit cumbersome, and we refer to section IV of ref. [5] for details. The result is that while this minimal set suffices for obtaining hierarchical solutions for both $\left\langle\chi_{u}\right\rangle$ and $\left\langle\chi_{d}\right\rangle$, only one nontrivial mixing angle $\left(\theta_{23}\right)$ is generated, which also implies that there are no sources of $\mathrm{CP}$ violation in the ground state for the mixing matrix $\langle K\rangle$.

\subsection{Mass hierarchies, CKM mixings and $\mathrm{CP}$ violation}

Generating the other two nonvanishing mixing angles $\left(\theta_{12}\right.$ and $\left.\theta_{13}\right)$ requires at least one additional multiplet transforming in the fundamental of $S U(3)_{Q}: Z_{Q_{2}} \sim(\mathbf{3}, \mathbf{1}, \mathbf{1})$. This can be intuitively understood by observing that four independent "directions" in L-handed flavour space $\left(Z_{Q_{1}}, Z_{Q_{2}} Z_{u}\right.$, $Z_{d}$ ) constitute the minimum number required to define three relative "angles". While the expression for the full $\mathscr{G}_{\mathscr{F}}$ invariant potential becomes a bit involved, many terms are flavour irrelevant, and understanding the dynamical action of the relevant invariants is still a manageable task. This task has been carried out in [5]. Quite interestingly, we have found that the same field content that ensures three nonvanishing mixing angles, is also sufficient to ensure that at the potential minimum the vev $\langle K\rangle \sim V_{C K M}$ contains one CP violating phase. Let us stress, for the seek of clarity, that in the present case the fact that the potential minimum is $\mathrm{CP}$ violating has nothing to do with the notion of spontaneous CP violation. In fact, in our scenario the scalar potential violates CP from the start because of the presence of a certain number of physical complex phases (four, to be exact). The important point thus is that at the minimum there is no restoration of $\mathrm{CP}$ symmetry, something that could have happened if, by some accident, all the field vevs had flown towards real values.

\subsection{A numerical example}

The final verdict if spontaneous breaking of the flavour symmetry is able to account for the entire set of observables in the quark sector can eventually be settled only by means of numeri-

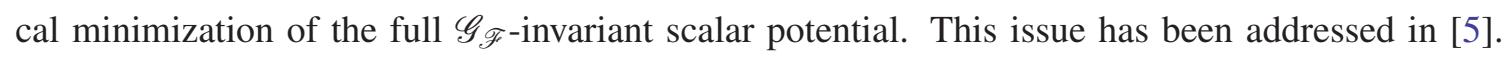
However, no attempts to perform multidimensional global fits to the SM observables where carried out, something that would have required a prohibitive amount of CPU time. The simpler approach followed in [5] was that of assuming a simple set of values for most of the flavour irrelevant parameters and then, by varying the remaining (crucial) ones, attempting to approximate the experimental 
values of the observables of the quark sector. Of course, carrying out successfully this procedure has been rendered feasible by a good understanding of the role of each term in the potential, an understanding that we have gained by inspection of several partial analytical results. An example of the type of results that can be obtained is given below. Since we have not reproduced here the (rather lengthy) expression for the most general $\mathscr{G}_{\mathscr{F}}$ invariant potential for the $Y$ and $Z$ fields, we also do not recopy the numerical values of the input parameters. Since it is a quite relevant point we mention, however, that all parameters have been taken to be $\mathscr{O}(1)$, with no hierarchies among them larger than $\mathscr{O}\left(10^{-1}\right)$. We have found input values satisfying the above conditions for which the resulting parameters of the SM quark sector are

$$
\begin{aligned}
\left\langle Y_{u}\right\rangle & =v_{u} \operatorname{diag}(0.0003,0.009,1.4), \quad\left\langle Y_{d}\right\rangle=v_{d} \operatorname{diag}(0.0007,0.02,1.2), \\
|\langle K\rangle| & =\left(\begin{array}{ccc}
0.974 & 0.223 & 0.027 \\
0.224 & 0.974 & 0.042 \\
0.017 & 0.046 & 0.999
\end{array}\right),
\end{aligned}
$$

with $J$ the Jarskog invariant [24]. From the fact that we have set $v_{d}=v_{u}$ from the start, it follows that the largest entries in $\left\langle Y_{u, d}\right\rangle$ are similar size. This does not constitute any real problem: the value of the $b$-quark mass can be easily suppressed by means of a $U(1)$ spurion vev, along the lines described in [3], or by extending the Higgs sector to a two doublets model with $\left\langle H_{d}\right\rangle \ll\left\langle H_{u}\right\rangle$.

\section{Conclusions}

In this contribution we have reported the attempt developed in the three papers $[3,4,5]$ to explain the values of the parameters of the SM quark sector (four mass ratios, three mixing angles and one $\mathrm{CP}$ violating phase) starting from the idea that the complete breaking of the quark flavour symmetry can result as the dynamical effect of driving a suitable scalar potential towards its minimum. We have identified the minimum set of multiplets in simple (fundamental and bifundamental) representations of the group needed to break $\mathscr{G}_{\mathscr{F}} \rightarrow 0$, and we have shown that this same set of fields is also sufficient to generate one weak $\mathrm{CP}$ violating phase. Besides the quantitative results, through this study we have gained important qualitative understandings of various mechanisms that might underlie some of the most puzzling features of the SM quark sector. We list them in what we think is their order of importance.

1. $K=V_{C K M} \approx I$. The interaction between the two Yukawa fields $Y_{u}$ and $Y_{d}$ tends to generate an exact alignment of their vevs in flavour space, resulting in $V_{C K M}=I$ [8]. If the interaction is repulsive $\left(\lambda_{u d}>0\right)$ the alignment occurs when the eigenvalues of the two matrices are ordered by size in an opposite way. When the interaction is attractive $\left(\lambda_{u d}<0\right)$ the alignment occurs when the ordering is the same. This second possibility is the one observed in nature. To generate three nonvanishing mixing angles, that is to (slightly) misalign $Y_{u}$ and $Y_{d}$ in all flavour directions, at least two other multiplets transforming under the L-handed factor $S U(3)_{Q}$ are needed. Their presence will induce perturbation in the exact alignment, but if the $Y_{u}-Y_{d}$ interaction is sufficiently strong, an approximate form $V_{C K M} \approx I$ will be maintained.

2. Yukawa hierarchies. Hierarchies between the different entries in $\left\langle Y_{u}\right\rangle$ and $\left\langle Y_{d}\right\rangle$ are seeded by taking for a subset of the dimensional parameters values somewhat smaller than the overall scale 
of the vevs: $\mu, v \sim v_{Y} / 10$. This can be justified by the fact that when these parameters are set to zero, the scalar potential gains some additional $U(1)$ symmetries. The initial (mild) suppression of some entries in $\left\langle Y_{u, d}\right\rangle$ can get amplified down to the observed values of the quark mass ratios by dynamical effects. Hierarchical Yukawa couplings can then be generated without strong hierarchies in the fundamental parameters.

3. $C P$ violation. Once the flavour symmetry is completely broken, generating the CKM CP violating phase does not require complicating further the model. The set of scalar multiplets needed to obtain $\mathscr{G}_{\mathscr{F}} \rightarrow 0$ ensures that several complex phases cannot be removed regardless of field redefinitions, and this ensures that the scalar potential contains $\mathrm{CP}$ violating terms. For generic values of these phases, a $\mathrm{CP}$ violating ground state for $V\left(Y_{q}, Z_{q}, Z_{Q_{1,2}}\right)$ is obtained.

Indeed, one could object that in our construction there are many more fundamental parameters than there are observables. This of course affects its predictivity, and in some respects also its elegance. We cannot object to such a criticism, but it is worth stressing that the proliferation of parameters is a result of the usual quantum field theory prescription for building renormalizable Lagrangians: we have identified the minimum number of multiplets needed to break completely $\mathscr{G}_{\mathscr{F}}$, and next we have simply written down the complete set of renormalizable operators allowed by the symmetry. After all, as it has been argued e.g. in [25], the apparent lack of simple relations between the observables of the quark sector might well be due to the fact that, as in our case, they are determined by a very large number of fundamental parameters.

Direct evidences of the scenario we have been studying might arise from the fact that if the flavour symmetry is global, then spontaneous symmetry breaking implies the presence of NambuGoldstone bosons that could show up in yet unseen hadron decays or in rare flavour violating processes. If the flavour symmetry is instead gauged, then to ensure the absence of gauge anomalies additional fermions must be introduced [15], and their detection could then represent a smoking gun for this type of models. All this remains, however, a bit speculative, especially because the theory provides no hint of the scale at which the flavour symmetry gets broken, and very large scales would suppress most, if not all, types of signatures.

\section{Acknowledgments}

It is a pleasure to thank J. R. Espinosa and C. S. Fong for their collaboration in developing this research project. This work is supported in part by the research grant number 2012CPPYP7 under the program PRIN 2012 funded by the Italian MIUR and by INFN under TAsP-LNF.

\section{References}

[1] C. Froggatt, H. B. Nielsen, Hierarchy of Quark Masses, Cabibbo Angles and CP Violation, Nucl.Phys. B147 (1979) 277.

[2] R. S. Chivukula, H. Georgi, Composite Technicolor Standard Model, Phys.Lett. B188 (1987) 99.

[3] E. Nardi, Naturally large Yukawa hierarchies, Phys.Rev.D 84 (2011) 036008 [arXiv: 1105.1770 ].

[4] J. R. Espinosa, C. S. Fong, E. Nardi, Yukawa hierarchies from spontaneous breaking of the $S U(3)_{L} \times S U(3)_{R}$ flavour symmetry?, JHEP 1302 (2013) 137 [arXiv: 1211.6428 ]. 
[5] C. S. Fong, E. Nardi, Quark masses, mixings, and CP violation from spontaneous breaking of flavor SU (3) ${ }^{3}$, Phys.Rev. D89 (3) (2014) 036008 [arXiv: 1307.4412 ].

[6] L. Michel, L. Radicati, Properties of the breaking of hadronic internal symmetry, Annals Phys.66 (1971) 758-783.

[7] N. Cabibbo, L. Maiani, in Evolution of particle physics, Academic Press 50 App. I (1970) 68-72.

[8] A. Anselm, Z. Berezhiani, Weak mixing angles as dynamical degrees of freedom, Nucl.Phys. B484 (1997) 97-123 [hep-ph/9605400]

[9] Z. Berezhiani, A. Rossi, Flavor structure, flavor symmetry and supersymmetry, Nucl.Phys.Proc.Suppl.101 (2001) 410-420 [hep-ph/ 0107054 ].

[10] Y. Koide, Phenomenological Meaning of a Neutrino Mass Matrix Related to Up-Quark Masses, Phys.Rev. D78 (2008) 093006 [arXiv: 0809 . 24 49].

[11] Y. Koide, Charged Lepton Mass Relations in a Supersymmetric Yukawaon Model, Phys.Rev. D79 (2009) 033009 [arXiv:0811.3470].

[12] Y. Koide, H. Nishiura, Yukawaon Model with $U(3) \times S_{3}$ Family Symmetries, Phys.Lett. B712 (2012) 396-400 [arXiv:1202.5815].

[13] T. Feldmann, M. Jung, T. Mannel, Sequential Flavour Symmetry Breaking, Phys.Rev. D80 (2009) 033003 [arXiv:0906.1523].

[14] M. Albrecht, T. Feldmann, T. Mannel, Goldstone Bosons in Effective Theories with Spontaneously Broken Flavour Symmetry, JHEP1010 (2010) 089 [arXiv: 1002 . 4798].

[15] B. Grinstein, M. Redi, G. Villadoro, Low Scale Flavor Gauge Symmetries, JHEP 1011 (2010) 067 [arXiv:1009.2049].

[16] R. Alonso, M. Gavela, L. Merlo, S. Rigolin, On the scalar potential of minimal flavour violation, JHEP1107 (2011) 012 [arXiv: 1103.2915$].$

[17] I. de Medeiros Varzielas, Non-Abelian family symmetries in Pati-Salam unification, JHEP1201 (2012) 097 [arXiv:1111.3952].

[18] R. N. Mohapatra, Gauged Flavor, Supersymmetry and Grand Unification, AIP Conf.Proc. 1467 (2012) 7-14.

[19] R. Alonso, M. Gavela, G. Isidori, L. Maiani, Neutrino Mixing and Masses from a Minimum Principle, JHEP1311 (2013) 187 [arXiv:1306.5927].

[20] E. Nardi, Quark Yukawa pattern from spontaneous breaking of flavour $S U(3)^{3}$, [arXiv:1503.01476].

[21] C. S. Fong, E. Nardi, Spontaneous Breaking of Flavor Symmetry Avoids the Strong CP Problem, Phys.Rev.Lett. 111 (6) (2013) 061601 [arXiv: 1305 . 1627].

[22] H. Georgi, A. Pais, Natural Stepwise Breaking of Gauge and Discrete Symmetries, Phys.Rev.D16 (1977) 3520.

[23] S. R. Coleman, E. J. Weinberg, Radiative Corrections as the Origin of Spontaneous Symmetry Breaking, Phys.Rev.D7 (1973) 1888-1910.

[24] C. Jarlskog, Commutator of the Quark Mass Matrices in the Standard Electroweak Model and a Measure of Maximal CP Violation, Phys.Rev.Lett.55 (1985) 1039.

[25] L. F. Duque, D. A. Gutierrez, E. Nardi, J. Norena, Fermion mass hierarchy and non-hierarchical mass ratios in $S U(5) x U(1)(F)$, Phys.Rev. D78 (2008) 035003 [arXiv : 0804.2865 ]. 